\title{
Fatigue size effect due to defects in an AA7050 alloy
}

\author{
Foued Abroug ${ }^{1-2}$, Etienne Pessard ${ }^{1, *}$, Guénaël Germain ${ }^{1}$ and Franck Morel $^{1}$ \\ ${ }^{1}$ LAMPA, Arts et Métiers ParisTech Angers, 2 Bd du Ronceray, 49035 Angers, France \\ ${ }^{2}$ LaMCoS, INSA -Lyon, 20 Avenue Albert Einstein, 69621 Villeurbanne, France
}

\begin{abstract}
One objective of this project is to propose a fatigue design approach that is able to account for a large range of machining surface defects and different component sizes and geometries. Due to the huge size difference between a typical fatigue specimen and large aircraft components it was first necessary to confirm if a size effect can indeed be observed. This was done by introducing different numbers of artificial surface defects on smooth specimens. The material investigated is a 7050 Aluminium alloy (Al $\mathrm{Zn6CuMgZr}$ ). Plane bending specimens both without and with artificial hemispherical surface defects were tested. The number of defects was varied from 1 to 44 defects per specimen and the defect size ranged from $60 \mu \mathrm{m}$ to $800 \mu \mathrm{m}$ in diameter. The test results allow the characterization of both the defect effect and scale effect on the fatigue response of the material. A probabilistic approach based on the weakest link concept together with a proper fatigue crack initiation criterion is used to account for the stress distribution and the size of the highly stressed volume. Predictions using FE simulations show a good agreement with experimental results and illustrate the importance of taking the scale effect into account in HCF.
\end{abstract}

\section{Introduction}

The effect of component size on fatigue strength is a well know problem in fatigue and has been investigated by many researchers since many years. Pogoretskii and Karpenko in 1965 [1] observed for example a high fatigue strength decreasing with the specimen length in rotating pure bending on a $37 \mathrm{Cr} 4$ steel (figure 1).

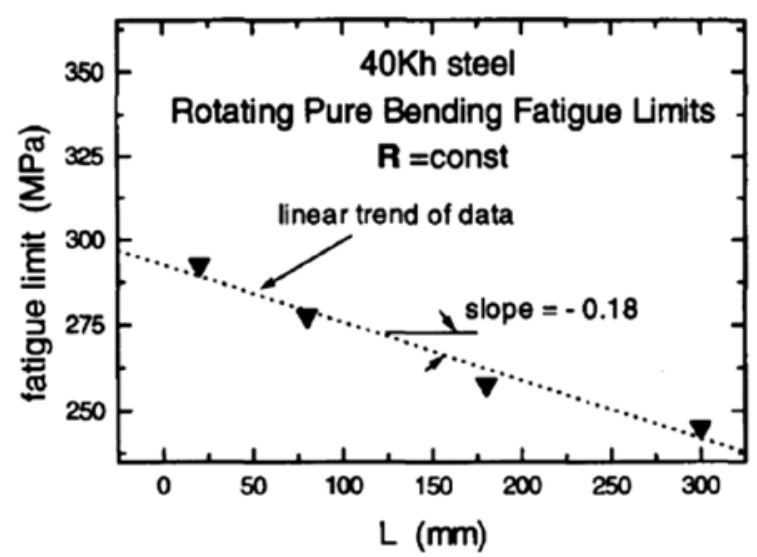

Fig. 1. Evolution of the fatigue strength in function of the specimen length [1]

The drop in the fatigue strength that is observed with increasing volume is very often explained by an increase in the probability of finding a critical defect or a weak zone as the volume of highly stressed material increases.

The impact of defect size on the fatigue strength is also well known and the Kitagawa-Takahashi diagram [2] is a useful tool to design material containing defects (see figure 2). The Kitagawa-Takahashi diagram in this case differed from his initial definition and the defect are considered as initial cracks.

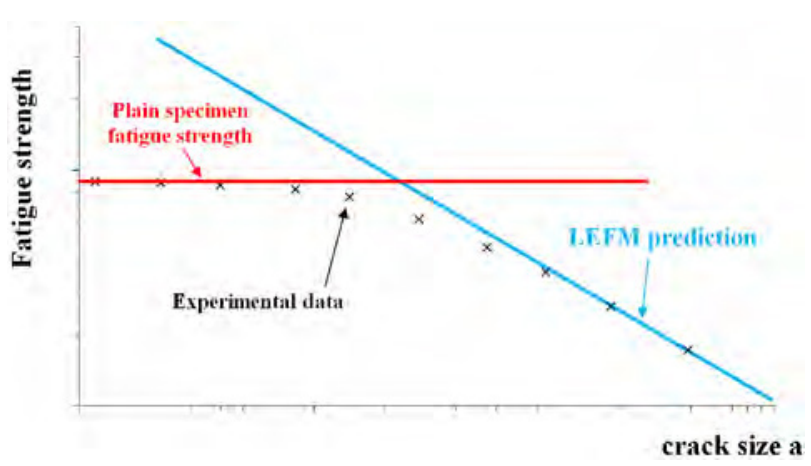

Fig. 2. Schematic representation of the Kitagawa-Takahashi diagram

The premise of this work is that these two phenomena are linked. That is, in a uniform stress field, without the presence of notches, it is really the defect size and the number of defects that control the stressed volume. Therefore, the Kitagawa-Takahashi diagram can be justified as an illustration of the scale effect.

In the present paper, the material and the experimental procedure will be firstly presented. Plane bending results with specimens both without and with artificial hemispherical surface defects to characterize both the defect effect and scale effect will be discussed. Then the observed specific size effect will be modeled by using Finite Element simulations together with the weakest link concept. 


\section{AA7050-T7451 alloy, experimental procedure and results}

\subsection{Material}

The material under investigation is the $\mathrm{Al} \mathrm{Zn} 6 \mathrm{CuMgZr}$ Aluminum alloy, also called AA 7050. The composition is presented in Table 1. Specimens are machined out of a $30 \mathrm{~mm}$ thick sheet in the rolling direction. The laminate operation creates a particular microstructure usually called "pan cake" microstructure with elongated grains in the rolling direction (from 5 to $300 \mu \mathrm{m}$ ) and thinner in the transverse direction (and 5 to $50 \mu \mathrm{m}$ in depth) (See Fig.3).

Table1. Chemical composition of the AA7050-T7451 alloy, in weight percentage.

\begin{tabular}{|c|c|c|c|l|l|l|l|l|}
\hline Element & $\mathrm{Zn}$ & $\mathrm{Cu}$ & $\mathrm{Mg}$ & $\mathrm{Zr}$ & $\mathrm{Ti}$ & $\mathrm{Si}$ & $\mathrm{Fe}$ & $\mathrm{Mn}$ \\
\hline Weight $\%$ & 6.03 & 2.22 & 1.85 & 0.10 & 0.039 & 0.038 & 0.098 & 0.01 \\
\hline
\end{tabular}

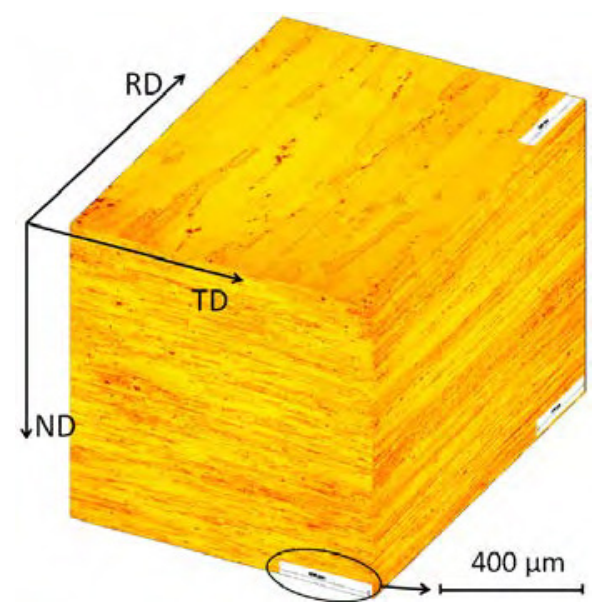

Fig. 3. AA7050-T7451 alloy a) Rolling direction, b) long transverse direction, c) short transverse direction

Different types of intermetallic particles are present at the grain boundaries and in the recrystallized grains (Mg2S i, Al7Cu2Fe, MgZn2, AlCuZn, etc...). These particles are brittle and can be at the origin of crack initiation under fatigue loading [3,4]. Monotonic tests show that the monotonic properties are slighty marked. The average Yield Strength and Ultimate Tensile Strength are respectively: $\sigma_{\mathrm{y}}=460 \mathrm{MPa}, \sigma_{\mathrm{UTS}}=556 \mathrm{MPa}$. The microhardness is also constant in the sheet depth with a value around $157 \mathrm{Hv} / 0.5$

\subsection{Fatigue tests conditions}

All the fatigue tests are carried out under fully reversed plane bending $(\mathrm{R}=-1)$ using a resonant testing machine at room temperature and ambient air at a frequency around $75 \mathrm{~Hz}$. The details of the machine and the geometry used are presented in Fig.4.

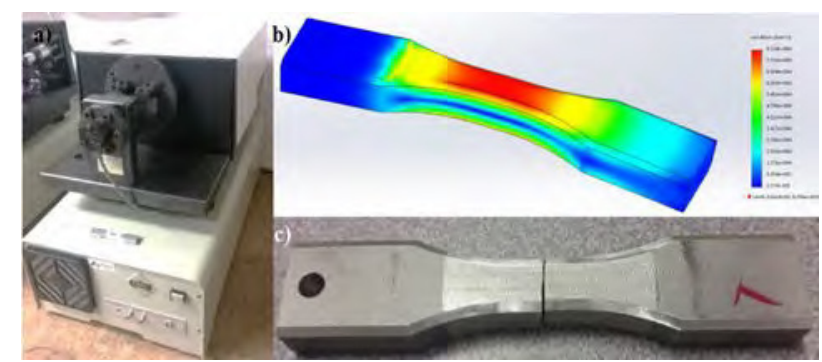

Fig. 4. AA7050-T7451 alloy a) Rolling direction, b) long transverse direction, c) short transverse direction

The endurance limit is determined at $2 \times 10^{6}$ cycles using Staircase or Locati methods. Stress value is decreased and increased by $10 \mathrm{MPa}$ step for Staircase and Locati method.

Due to the high number of cycles applied on each steps, fatigue limit is estimated as the mean stress value between the final loading step leading to failure and the step before. Specimens are polished and artificial defects of 6 different sizes are introduced by micro-drilling technique (Ø60, 100, 200, 400, 600, and Ø800 $\mu \mathrm{m})$. After machining, defects are scanned with a 3D BRUKER type profilometer (see Fig. 6). As it can be seen in Fig. 5, defect depths and diameters are carefully adjusted and controlled.

In order to characterize the scale effect, 2-3 specimens per defect size are tested with 1 and 22 defects per specimen. In addition, 3 specimens are tested in presence of 44 defects of $\varnothing 400 \mu \mathrm{m}$. To complete this large experimental campaign also presented in [5], a specimen containing 30 defects $\varnothing 200 \mu \mathrm{m}$ and one defect $\varnothing 400 \mu \mathrm{m}$ is tested.

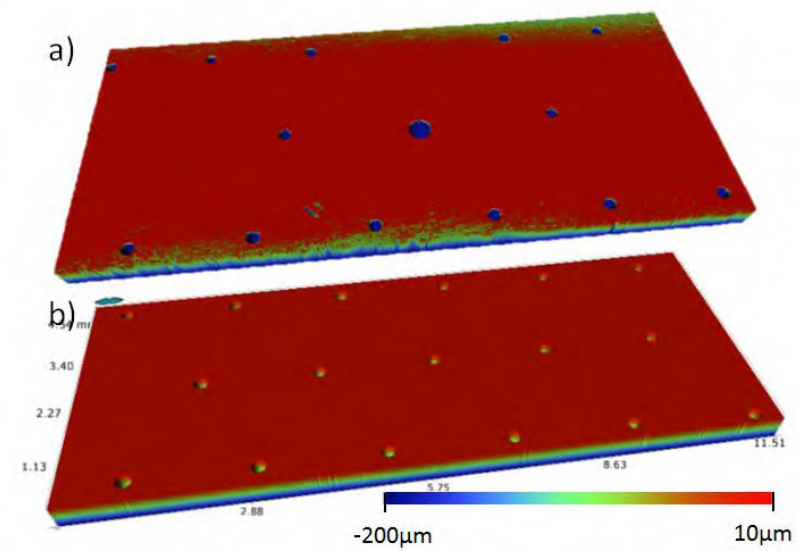

Fig. 5. Surface topography of the surface specimen a) face I containing 13 defects $\varnothing 200 \mu \mathrm{m}$ and one defect $\varnothing 400 \mu \mathrm{m}$, b) face II containing 17 defects $\varnothing 200 \mu \mathrm{m}$

\subsection{Fatigue results}

All fatigue tests are summarizing on Kitagawa Takahashi diagram in Fig. 6 and on the Table 1. On the Table 2, the standard deviation is estimated only for the 3 fatigue limits characterized via the Staircase approach. 


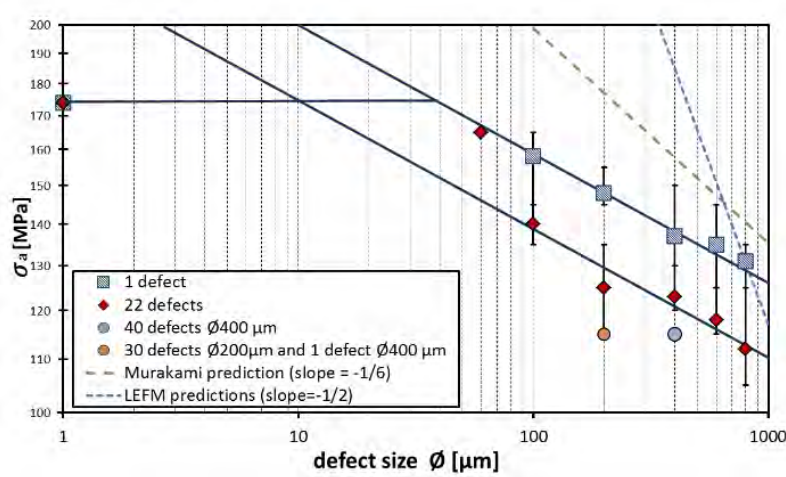

Fig. 6. Kitagawa-Takahashi diagram, AA7050alloy: experimental results for different defect size and number.

Table 2 : Fatigue test results

\begin{tabular}{|c|c|c|c|}
\hline $\begin{array}{c}\text { Defect } \\
\text { size }(\boldsymbol{\mu m})\end{array}$ & $\begin{array}{c}\text { Number } \\
\text { of defect }\end{array}$ & $\begin{array}{c}\text { Number of } \\
\text { specimens }\end{array}$ & $\begin{array}{c}\text { Fatigue limit at } \\
\mathbf{2 \times 1 0 ^ { 6 }} \text { cyles (MPa) }\end{array}$ \\
\hline \multirow{2}{*}{0 -Polished } & 0 & 15 & $174 \pm 5.3$ \\
\hline$\varnothing 60$ & 22 & 2 & 165 \\
\hline \multirow{2}{*}{$\varnothing 100$} & 1 & 3 & 158 \\
\cline { 2 - 4 } & 22 & 3 & 140 \\
\hline \multirow{2}{*}{$\varnothing 200$} & 1 & 3 & 148 \\
\cline { 2 - 4 } & 22 & 3 & 125 \\
\hline \multirow{2}{*}{$\varnothing 400$} & 1 & 10 & $137 \pm 9.5$ \\
\cline { 2 - 4 } & 22 & 10 & $123 \pm 5.3$ \\
\cline { 2 - 4 } & 44 & 3 & 115 \\
\hline \multirow{2}{*}{$\varnothing 600$} & 1 & 3 & 135 \\
\cline { 2 - 4 } & 22 & 3 & 118 \\
\hline \multirow{2}{*}{$\varnothing 800$} & 1 & 3 & 131 \\
\cline { 2 - 4 } & 22 & 3 & 112 \\
\hline \multirow{2}{*}{30 defects $\varnothing 200 \mu \mathrm{m}$ and } & 1 & 115 \\
\hline \multicolumn{2}{|c|}{1 defect $\varnothing 400 \mu \mathrm{m}$} & 1 & \\
\hline
\end{tabular}

In presence of a single defect, above a critical defect size, the fatigue decreases progressively and follows a straight line in the $\log \left(\sigma_{\mathrm{a}}\right)-\log$ (defect-size) diagram (see Fig. 6). This behaviour is commonly known as the Kitagawa effect [2]. As shown in Fig. 6, the slope of decrease is the same in case of 1 and 22 defects and around $(-1 / 10)$. Note that the defect sensitivity is lower compared to the Murakami criterion [6] and Linear Elastic Fracture Mechanics with slopes (-1/6) and (-1/2) respectively.

As shown on the Kitagawa-Takahashi diagram (Fig. 6) the fatigue limit decreases with the number of introduced defects. A drop of $20 \mathrm{MPa}$ is observed when the number of defects is multiplied by 22. For specimens containing $\varnothing 400 \mu \mathrm{m}$ defects the fatigue limit decreasing seems to be less pronounced when the number of defects goes from 22 to 40 compare to 1 to 22 .

The effect of the defect number on the fatigue limit can be explained by the scale effect. When increasing the number of defects, the highly stressed volume increases and the probability of finding a heterogeneity (i.e particle) or an unfavorably oriented grain increases.
Hence, crack initiation is more likely to occur at a lower stress value than in the case with a single defect.

The fatigue test result on the specimen containing 30 defects $\varnothing 200 \mu \mathrm{m}$ and one defect $\varnothing 400 \mu \mathrm{m}$ illustrates that the size effect controls the impact of the defect size and the number on fatigue (see Fig.7 and Fig.8). For this particular specimen, initiation doesn't start from the largest defect $(\varnothing 400 \mu \mathrm{m})$ but from one of the thirty smallest defect $(\varnothing 200 \mu \mathrm{m})$. Even if the $\varnothing 200 \mu \mathrm{m}$ defects are smallest, the thirty $\varnothing 200 \mu \mathrm{m}$ defects create a largest highly stressed volume compared to the single defect $\varnothing 400 \mu \mathrm{m}$. The probability to find a heterogeneity around a $\varnothing 200 \mu \mathrm{m}$ defect is therefore higher and initiation does not start from the largest defect.

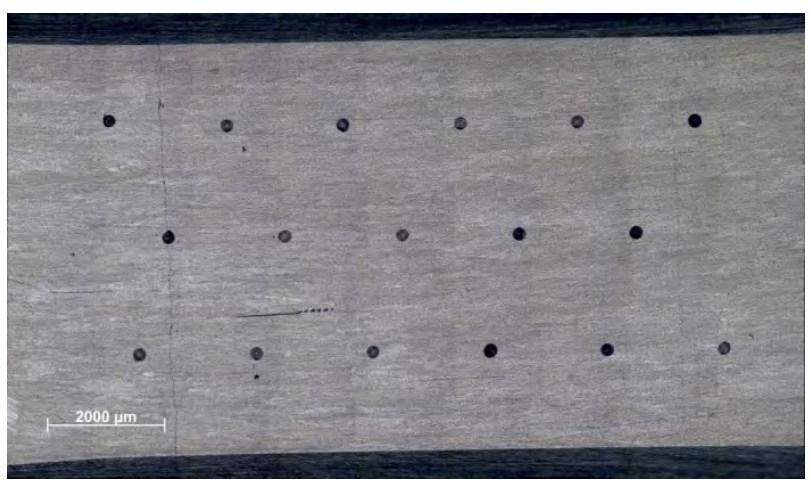

Fig. 7. Surface specimen after fatigue test showing a crack starting from a $\varnothing 200 \mu \mathrm{m}$ defect on the face II on the specimen containing 30 defects $\varnothing 200 \mu \mathrm{m}$ and one defect $\varnothing 400 \mu \mathrm{m}$ (see figure 5).

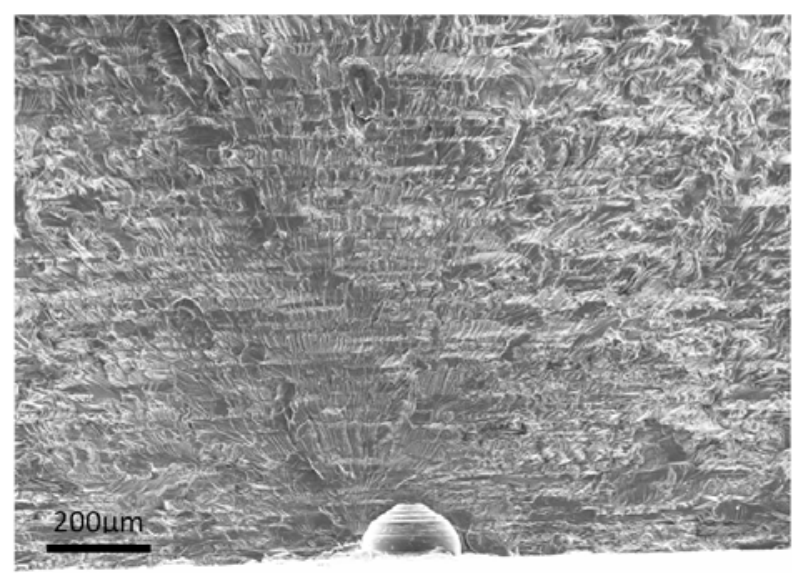

Fig. 8. Failure surface of the specimen containing 30 defects $\varnothing 200 \mu \mathrm{m}$ and one defect $\varnothing 400 \mu \mathrm{m}$ : initiation start from a $\varnothing 200 \mu \mathrm{m}$ defect.

\section{Probabilistic fatigue criterion}

\subsection{Probabilistic framework}

This section is about the modelling of the crack initiation damage mechanism in a polycrystalline aggregate containing geometric defects. 
As a general rule most fatigue criteria can be defined by an equivalent mechanical quantity (i.e. stress, strain, energy, etc.), which is compared to a threshold quantity, for a given number of cycles. Crack initiation is subsequently predicted if the equivalent quantity is greater than or equal to the threshold quantity. In the following, for convenience, these will be referred to stress quantities. Hence, the condition for crack initiation is defined by:

$$
\sigma_{e q} \geq \sigma_{t h}
$$

In order to take into account the inherent stochastic nature of the fatigue phenomenon it is proposed, as part of the modelling framework, to use a two parameter Weibull distribution [7,8] to describe the threshold stress $\sigma_{t h}$

$$
f_{0}\left(\sigma_{t h}\right)=\frac{\mathrm{m}}{\sigma_{0}}\left(\frac{\sigma_{t h}}{\sigma_{0}}\right)^{m-1} \exp \left\{-\left(\frac{\sigma_{t h}}{\sigma_{0}}\right)^{m}\right\}
$$

where $\sigma_{0}$ is the scale parameter and $\mathrm{m}$ is the shape parameter (or the Weibull exponent) used to reflect the scatter associated with the threshold stress and hence the fatigue limit.

The probability of microcrack initiation corresponds to the probability of finding a threshold stress $\sigma_{\text {th }}$ that is less than the applied equivalent stress $\sigma_{\text {eq. }}$. This can be expressed as:

$$
P_{F_{0}}=P\left(\sigma_{t h}<\sigma_{e q}\right)=\int_{0}^{\sigma_{e q}} f_{0}\left(\sigma_{t h}\right) d \sigma_{t h}
$$

Using the simple equation of Weibull's distribution function, the integration allows to express the failure probability as follows:

$$
P_{F_{0}}=1-\exp \left[-\left(\frac{\sigma_{e q}}{\sigma_{0}}\right)^{m}\right]
$$

To obtain the probability of crack initiation for the complete structure $\mathrm{P}_{\mathrm{F}}$, the weakest link theory is used [9]. The probability of survival of the structure $\left(1-\mathrm{P}_{\mathrm{F}}\right)$ is defined as the product of the survival probabilities of each elementary volume. The quantity $\mathrm{V}_{0}$ corresponds to a reference volume and $\mathrm{V}_{\Omega}$ is the total volume considered.

$$
1-P_{F}=\prod_{V_{\Omega}}\left(1-P_{F_{0}}\right)
$$

Hence the probability of survival of the complete structure is given by:

$$
P_{F}=1-\exp \left[-\frac{1}{V_{0}} \iiint_{V_{\Omega}}\left(\frac{\sigma_{e q}}{\sigma_{0}}\right)^{m} d V\right]
$$

Introducing the surface stress heterogeneity factor on the component surface $\mathrm{H}_{\mathrm{m}}[10]$ :

$$
H_{m}=\frac{1}{V_{\Omega}}\left[\iiint_{V_{\Omega}}\left(\frac{\sigma_{e q}}{\sigma_{\max }}\right)^{m} d V\right]
$$

with $\sigma_{\max }$ the maximum equivalent stress on the volume considered. The expression for the failure probability of the total structure becomes:

$$
P_{F}=1-\exp \left[-\frac{V_{\Omega}}{V_{0}} H_{m}\left(\frac{\sigma_{\max }}{\sigma_{0}}\right)^{m}\right]
$$

In order to simplify the expression and to make a link with the high cycle fatigue limit, the macroscopic fatigue limit $\sigma_{\mathrm{a}}$ can be expressed via the following expression:

$$
\sigma_{a}=\frac{\sigma_{0}}{K_{t_{\max }}}\left[\frac{V_{0}}{V_{\Omega}} \frac{\ln \left(\frac{1}{1-P_{F}}\right)}{H_{m}}\right]^{1 / m}
$$

Where $\mathrm{K}_{\mathrm{tmax}}$ is the maximal equivalent stress concentration factor which corresponds to the maximal equivalent stress divided by the nominal equivalent applied stress.

In the case of polished specimen and a simple uniaxial loading, the surface stress heterogeneity factor $\mathrm{H}_{\mathrm{m}}$ could be easily obtained by an analytical calculation [10]. In our study, the surface stress field due to hemispherical defect is more complex and numerical finite element simulation must be conducted to assess this quantity.

\subsection{Finite element simulation}

To simplify the calculation, the specimen is divided in different cells. Using the weakest link theory (Eq 5) allows calculating the failure probability of the entire specimen from the products of the failure probability of cells containing or not a defect.

The finite element simulation is performed on a cube containing a hemispherical defect on its upper surface (see Fig. 9). Calculation is conducted in order to assess the stress in each element. Elements are tetrahedral with reduced integration and convergence of calculation results is verified with the chosen size of elements. The material is supposed to be homogeneous and an elastic behaviour is used. The potential residual stresses are also neglected. The boundary conditions are as follows: 


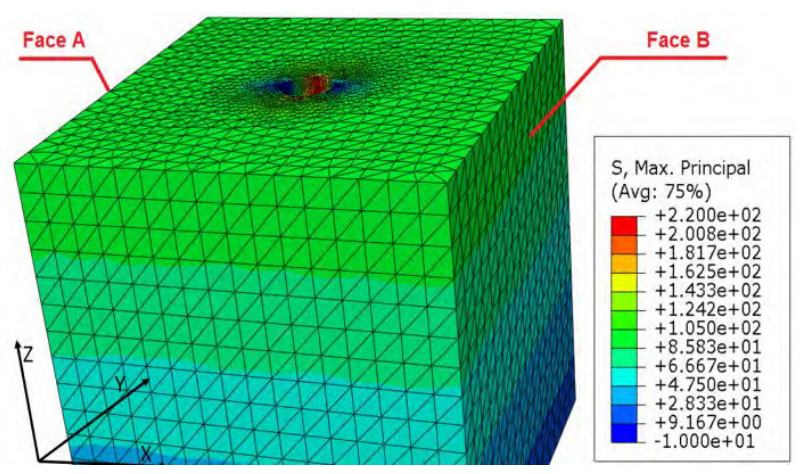

Fig. 9. FE simulation of a cube with hemispherical defect under bending loading

- Displacement $\mathrm{Ux}=0$ along the plane $\mathrm{X}=0$ (surface $\mathrm{A})$.

- Displacement $\mathrm{Uy}=0$ along the line $\mathrm{X}=0, \mathrm{Y}=0$.

- Displacement $\mathrm{Uz}=0$ at the point $\mathrm{X}=0, \mathrm{Y}=0, \mathrm{Z}=0$.

A bending pressure function representative of experimental bending loading is applied along the surface B (see Fig. 9).

It is well known in fatigue that initiation in metallic alloys mostly occurs in the surface or subsurface. Failure surface observations show for specimen with artificial defects that intermetallic particles are mostly localized in the first $50 \mu \mathrm{m}$ in depth around the defect. In our failure probability calculation only the first $50 \mu \mathrm{m}$ in depth will therefore be considered (see Fig. 10).

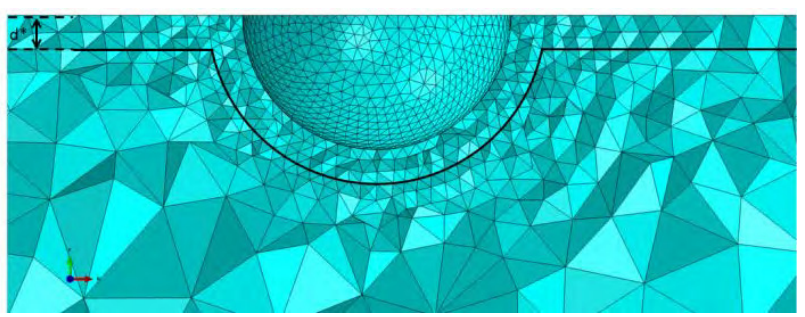

Fig. 10. Illustration of the $d^{*}=50 \mu \mathrm{m}$ parameter in the Finite Element Model.

To identify the two model parameters $\mathrm{m}$ and $\sigma_{0}$ that defines the Weibull distribution of the equivalent stress threshold, the staircase results obtained on the specimens containing $\varnothing 400 \mu \mathrm{m}$ defect are used. Finite Element Simulation of the bending test on a specimen containing a hemispherical defect of $\varnothing 400 \mu \mathrm{m}$ has been conducted with different value of $\mathrm{m}$ to calculate the stress heterogeneity factor. In the simple case of a bending loading study in this works the maximal principal stress is considered. The maximal stress concentration factor $\mathrm{K}_{\text {tmax }}$ is the same for the different defects size and is equal to 2.2 .

The scale parameter $\sigma_{0}$ is then calculated using Eq. (9). The couple of value $\left(\mathrm{m}, \sigma_{0}\right)$ that provides the lower error value between predictions and all experimental results is the couple $\left(\mathrm{m}=22 ; \sigma_{0}=226 \mathrm{MPa}\right)$.

\subsection{Predictions}

The predictions using the developed approach are in rather good agreement with the experimental results. The criterion correctly predicts the fatigue strength of the polished samples and the effect of the size and number of defect. The maximal error is observed for the specimen containing 22 defects of $\varnothing 60 \mu \mathrm{m}$. The approach for the smallest defect size is conservative and overestimates the material sensitivity to the defects.

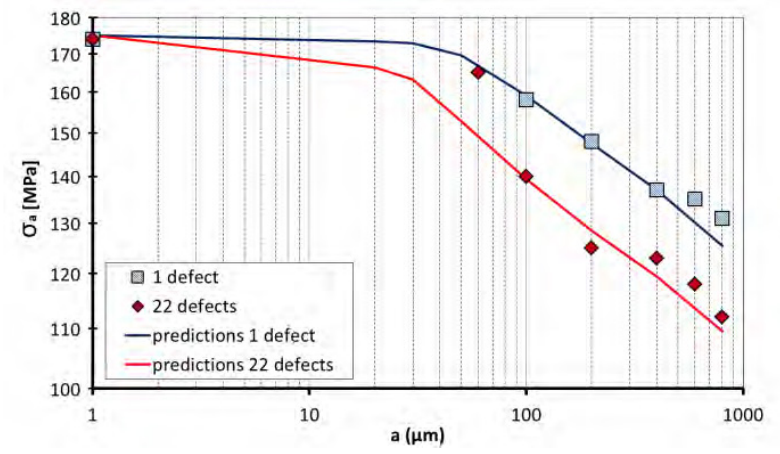

Fig. 11. Kitagawa diagram: comparison between experimental results and fatigue criterion predictions

The Fig. 12 shows the evolution of the fatigue strength with the number of defects.

Predictions show a good agreement with experimental results. Furthermore, it is shown that the decrease in fatigue limit slows down with the increase of defect number. The probabilistic model predicts that after a number of defects around 30, fatigue limit decrease is no longer significant. This tendency is usual for statistical size effect $[11,12]$.

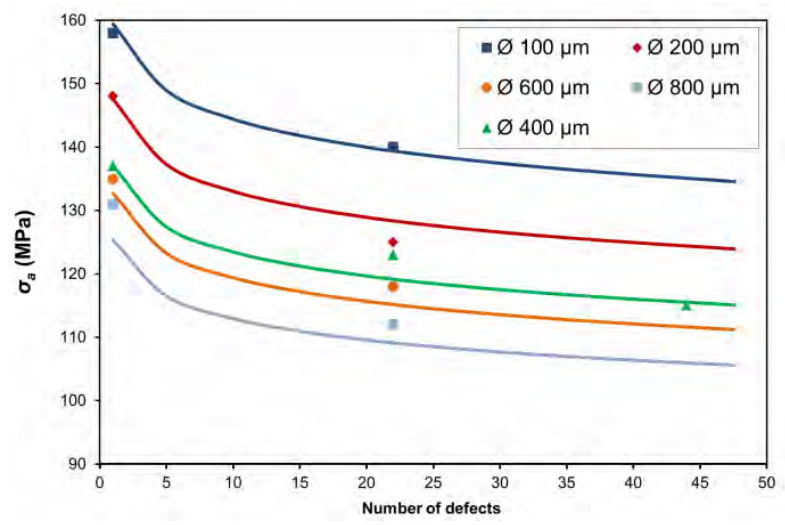

Fig. 12. Evolution of the fatigue limit with the number of defects comparison between experimental and predictions

It is not possible to compare in the Kitagawa diagram Fig. 11 and on the diagram Fig. 12 the result obtained on the last specimen containing 30 defects $\varnothing 200 \mu \mathrm{m}$ and one defect $\varnothing 400 \mu \mathrm{m}$. Based on the expression (Eq.9) of the fatigue strength using the criterion developed, the evolution of the fatigue strength in function of the product of $\mathrm{K}_{\text {tmax }} \mathrm{H}_{\mathrm{m}}{ }^{1 / \mathrm{m}}$ is plotted in Fig.13. In our case with a $\mathrm{Kt}_{\max }$ equal to 2.2 for all specimens, this figure illustrates that the number and the size of defects control the stress heterogeneity factor $\mathrm{H}_{\mathrm{m}}$. It is clear in this 
figure that a single defect $\varnothing 400 \mu \mathrm{m}$ is less critical in fatigue that 20 defects $\varnothing 200 \mu \mathrm{m}$ and so that 30 defects $\varnothing 200 \mu \mathrm{m}$. The same conclusion is observed between one defect $\varnothing 200 \mu \mathrm{m}$ and 20 defects $\varnothing 100 \mu \mathrm{m}$.

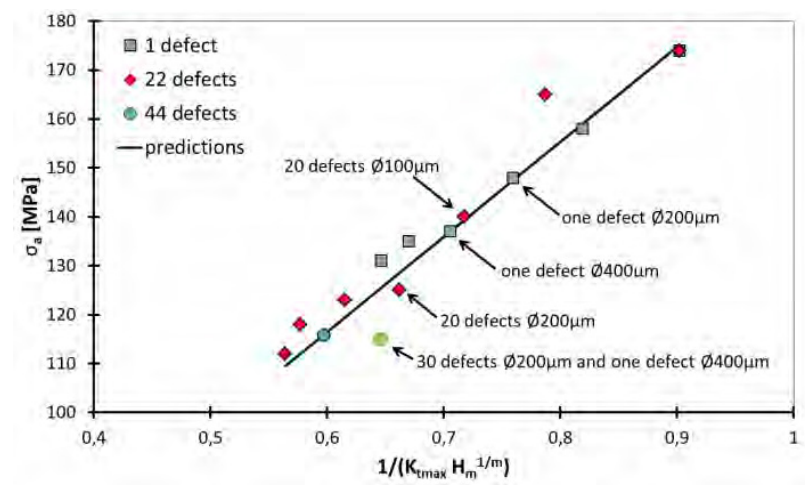

Fig. 13. Evolution of the fatigue limit in function of the $\left(\mathrm{K}_{\mathrm{tmax}}\right.$ $\left.\mathrm{H}_{\mathrm{m}}^{1 / \mathrm{m}}\right)^{-1}$ : comparison between experimental results and predictions

The quantity $\mathrm{K}_{\mathrm{tmax}} \mathrm{H}_{\mathrm{m}}{ }^{1 / \mathrm{m}}$ seems to be a relevant parameter to quantify the impact of defects or surface topography on the fatigue limit. The Figure 13 also illustrates that, knowing the $\mathrm{K}_{\mathrm{tmax}} \mathrm{H}_{\mathrm{m}}{ }^{1 / \mathrm{m}}$ generated by any surface defect population allows predicting the high cycles fatigue strength of the component using the proposed approach

\section{Conclusions}

The main conclusions of this work are:

- A vast experimental plane bending fatigue campaign on specimens containing different defect size and number have been conducted.

- The Kitagawa diagram obtained shows a low sensitivity of the AA7050 alloy to the defect size compared to usual fatigue criterion approach (LEFM and Murakami).

- The studied material shows a high sensitivity to the number of defects.

- An approach based on Weibull's distribution law and the weakest link concept is proposed to take into account the high stressed volume size of each specimen. It allows to accurately predict the fatigue limit for all specimens (containing or not a single or several defects).

Beyond the results obtained on the AA7050 alloys, these works illustrate the importance of taking the scale effect into account when designing in fatigue. Usually only the impact of the largest defect is considered. However for some materials and manufacturing processes, the defect density on the surface or in the core is of great importance in fatigue.

\section{Acknowledgements}

This work was carried out within the FUI QUAUSI project with the support of industrial (Dassault Aviation, Europe Technologie, Figeac Aéro, Mecachrome, Spring Technologie, Precise, GEBE2, CETIM) and academic partners (LSN2-IUT Carquefou).

\section{References}

1. R. G. Pogoretskii and G. V Karpenko, FizikoKhimicheskaya Mekhanika Materialov, pp. 1, 9094, (1965).

2. H. Kitagawa, and S. Takahashi, Metalspark, Ohio, p. 627-731, (1976).

3. M. Shahzad et al., Procedia Engineering, 2, pp 1015-1024 (2010).

4. V. K. Gupta and S. R. Agnew, Int J Fatigue, Vol 33 (2011).

5. F. Abroug et al., Int J Fatigue 110 81-94 (2018)

6. Y. Murakami Metal fatigue: effects of small defects and nonmetallic inclusions. Elsevier; (2002).

7. W. Weibull A statistical theory of the strength of materials. Roy. Swed. Inst. Eng. Res. Report 151; (1939).

8. Weibull W. ASME J Appl Mech 1951;18:293-7 (1951).

9. A.M. Freudenthal and H. Liebowitz, editor. Fracture, vol. 2. New York: Academic Press; p. 591-619 (1968).

10. L. Flaceliere and F. Morel. Fatigue Fract Eng Mater Struct 27(12):1123-35, (2004).

11. I.V. Papadopoulos. G, Lindley TC, editors. Multiaxial fatigue and design, ESIS 21. London: Mechanical Engineering Publications; p. 349-64. (1996).

12. T. Delahay and T. Palin-Luc Int J Fatigue 28:47484, (2006). 